\title{
Neurobiologia, neurorehabilitación y neurorestauración de la marcha del adulto mayor: conceptos recientes
}

\author{
Jaime Bayona-Prieto, Pedro Antonio Calero Saa y Marco Antonio Chaves García \\ Universidad de Boyacá, Tunja, Colombia
}

Palabras clave: marcha; longevidad; neurorehabilitación; neurorestauración.

Resumen. La población mundial ha crecido de manera exponencial en el siglo veintiuno, incluyendo la población longeva. Afortunadamente, los avances realizados, a la fecha, demuestran que el envejecimiento normal no es una enfermedad. Los cambios demográficos recientes, sin embargo, requieren de fundamentos biológicos actualizados que permitan realizar intervenciones oportunas, basadas en evidencias neurocientíficas válidas. Desafortunadamente, aún se desconoce un gran número de aspectos neurobiológicos asociados a la marcha de la población longeva. Por tal motivo, se hace imperativo establecer, de manera integrada, los aspectos biológicos, clínicos y de neurorehabilitación más relevantes, relacionados con la marcha y la locomoción, durante el envejecimiento normal. Este trabajo llena dicho vacío conceptual y ofrece un nuevo modelo holístico, para comprender mejor la marcha del adulto mayor. Así mismo, dicho modelo incluye abordajes modernos de neurorehabilitación y neurorestauración, tendientes a mejorar la comprensión neurofisiátrica de la marcha del adulto mayor.

\footnotetext{
Autor de Correspondencia: Jaime Bayona-Prieto, Carrera 33 N 54-33, Bucaramanga, Colombia; Teléfono: +57-7- 6850500; Correo electronico: jaimebayonap@gmail.com.
} 


\title{
Neurobiology, neurorehabilitation and restorative neurology in the elderly: recent concepts
}

Invest Clin 2018; 59(1): 52 - 66

Keywords: gait; longevity; neurorehabilitation; restorative neurology.

\begin{abstract}
The world's population has grown exponentially in the twenty-first century. Such growth includes the oldest population. Fortunately, the advances made in recent years have shown that normal aging is not a disease. The recent changes in demographics require, however, to update the fundamentals of biology that allow timely interventions in old people that should be based in valid neuroscientific evidences. Unfortunately, there are still a number of neurobiological aspects associated to longevity that remain unknown. These facts require updating information that allows a deeper understanding of the biological, clinical and neurorehabilitation aspects related to normal aging, particularly gait and locomotion. This work fills the existing gap and offers a new holistic model to better understand gait and locomotion of healthy aged people. The model put together modern approaches in neurorehabilitation and neuorestauration looking to improve the neurophysiatric understanding of the gait of healthy aging.
\end{abstract}

Recibido: 01-06-2017 Aceptado: 05-10-2017

\section{INTRODUCCIÓN}

Estudios recientes han determinado que la humanidad llegará a ser una población con una alta prevalencia de individuos longevos (1), más pronto de lo planeado a finales del siglo pasado. Por ejemplo, en Colombia la proyección para el 2020 es que el 19\% de la población será longeva (2). Por esta razón, el conocimiento sobre el envejecimiento y los cambios que esto genera en el humano, son motivo de atención global. Dentro de estos cambios, se destaca aquellos que se dan en la marcha humana.
La marcha humana, es un modo de locomoción bípeda que permite deambular usando los miembros inferiores. La marcha se caracteriza por una sucesión de acciones de los pies llamadas apoyo unipodal y bipodal (3). Dichos apoyos se aprenden durante los primeros años de vida y se replican, adaptan o modifican durante el resto de la existencia del individuo $(1,4)$.

El adulto mayor efectúa la marcha de manera lenta y cautelosa que, aunque no es patológica, le permite conservar la estabilidad (5) al modificar la base de soporte (6). Desafortunadamente, en 
ocasiones dicha marcha se enmarca dentro de lo patológico por algunos profesionales de la salud. Por hechos como éste, se hace necesario que actualizar aspectos fundamentales de la marcha en el adulto mayor, con el fin de orientar al mejoramiento funcional de este grupo poblacional.

El objetivo de este trabajo es presentar una actualización narrativa de la marcha en adultos mayores sanos, con el fin de contribuir a la reorientación de las prácticas biomédicas actuales. Además, se presenta un modelo holístico y novedoso de la marcha, que ayudará a reorientar prácticas relacionadas con la comprensión y reaprendizaje de la marcha del adulto mayor, como una forma de neurorestauración de procesos "normales" o no patológicos en los humanos

\section{ASPECTOS BÁSICOS}

El envejecimiento del ser humano es un estado progresivo, continuo y complejo. En consecuencia, se debe analizar desde diversos ángulos, incluyendo el de la marcha la cual se torna lenta, logrando modificar la vitalidad y capacidad del adulto mayor para realizar la marcha $(1,2,7)$. Es claro que cada individuo es diferente y, por ende, su envejecimiento se debe abordar de manera particular $(3,8)$. Sin embargo, este proceso complejo de envejecimiento no es considerado por los profesionales de salud general, en particular por los médicos, durante la evaluación clínica del paciente.

De manera clásica, el adulto mayor realiza la marcha usando una postura bípeda, desplaza el cuerpo hacia adelante y ejecuta una acción selectiva muscular y particular de cada individuo. Con estas acciones busca optimizar sus energías de la mejor manera posible, de acuerdo a su particular condición física, a expensas de modificaciones plásticas de sus ejes neurales $(5-7,9)$.
Debido a lo anterior, la marcha tiende a ser inestable, sin que esto signifique que sea patológica $(6-8,10)$. Sin embargo, en un buen número de ocasiones, la marcha lenta se relaciona con deterioro de la calidad de vida $(7,11)$. Esto conduce al individuo longevo o a sus familiares y cuidadores a la búsqueda de orientación del personal de la salud. En ocasiones, un buen número de profesionales de la salud no cuentan con las herramientas apropiadas para realizar la mejor valoración posible. La evaluación médica de estos individuos requiere la participación de grupos multi e interdisciplinares de profesionales de la salud, quienes requieren comprender que muy posiblemente, son ellos quienes identificarán el declive funcional del adulto mayor $(6,12,13)$.

La evaluación de la marcha del adulto mayor requiere que los profesionales de la salud conozcan a cabalidad, los mecanismos que integran la propiocepción, la función vestibular y la función visual (13). El ingreso de la información proveniente de estos sistemas, y la integración apropiada de dichas funciones y sistemas, a nivel intracraneal e intraespinal, generará el alineamiento corporal a nivel del sistema motor, la locomoción y correspondiente estabilidad, acorde con la edad (8-11) (Fig.1). Cabe enfatizar que esta integración sensorimotora se adapta con el paso de los años, de manera plástica, a las necesidades del individuo a medida que este envejece. Esto le permite al sistema nervioso estar en constante aprendizaje para mantener la bipedestación y la marcha de la mejor forma posible (11-14).

La marcha se define como una sucesión de pasos (11). El paso es una sumatoria de fenómenos que se producen entre el apoyo de un talón y el apoyo sucesivo del talón contralateral, que se repite en ciclos (12). El ciclo de la marcha es el conjunto de acciones y fenómenos producidos en el organismo, desde el contacto del talón con una superficie, hasta el siguiente 
contacto del mismo talón con dicho plano de apoyo $(13,14)$. Estos ciclos presentan variantes en diversas etapas de la vida (15).

En adultos mayores, la marcha tiene características particulares (16). Por ejemplo, el adulto mayor camina con cautela, dando la impresión de caminar como si lo hiciera sobre una superficie inestable o resbalosa (17).

Factores corporales externos poco estudiados a la fecha, también influyen en la marcha del adulto mayor $(18,19)$, lo que ha dificultado el establecimiento de un protocolo único de neurorehabilitación de la marcha, en la población longeva $(15,20)$.

\section{ASPECTOS CLÍNICOS}

La velocidad de marcha del adulto mayor predice su tendencia a la fragilidad, rendimiento y funcionalidad (21). A los 60 años de edad, el $85 \%$ de la población tiene una marcha que se considera normal. Hacia los 75 años de edad, la velocidad de la marcha disminuye hasta en un $16 \%$ (22). En la población mayor de 80 años de edad, la velocidad de la marcha disminuye hasta en un 20\% (23). Desde los 85 años de edad, un $40 \%$ de la población realiza una marcha que se considera anormal $(17,24)$.

Se ha comprobado que los individuos que muestran disminución de la velocidad en la marcha, también presentan alteraciones

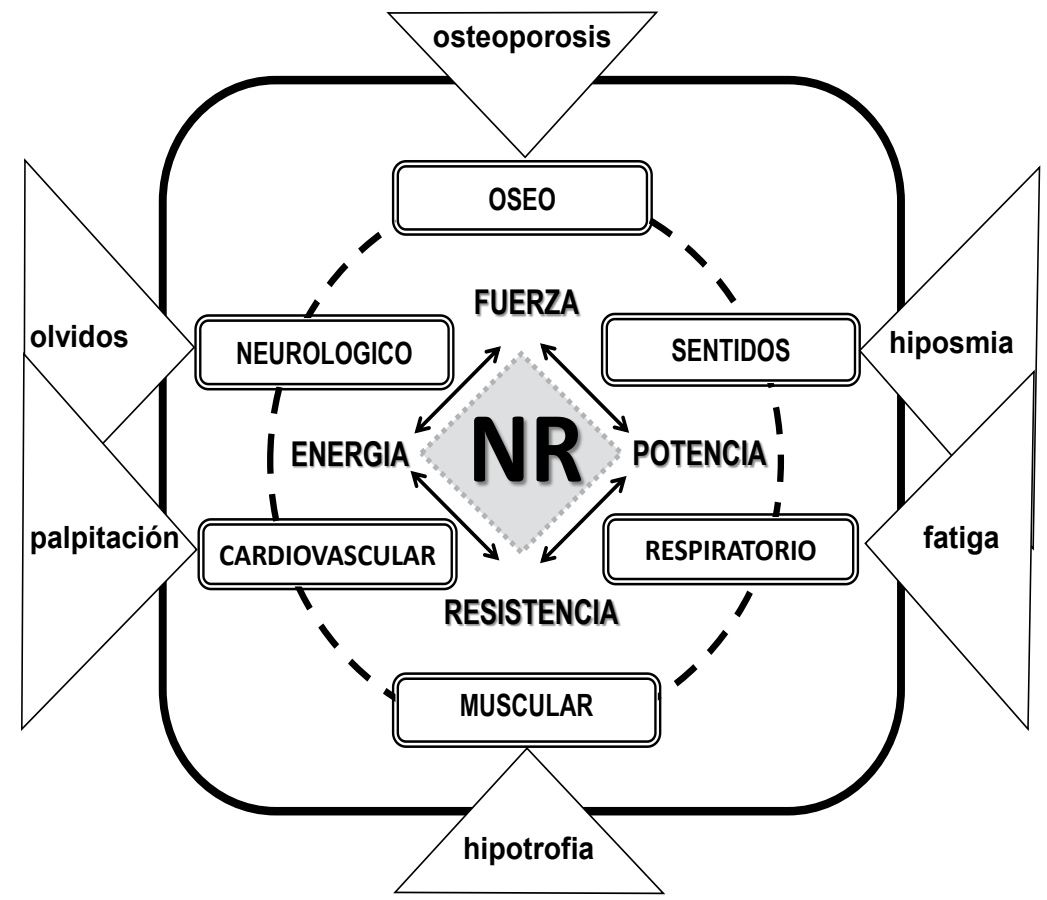

Fig. 1. Aspectos clinicos del envejecimiento. Alteraciones (triángulos) de la homeostasia (línea continua), debido al envejecimiento (circulo a trazos) de los sistemas corporales (rectángulos). Moduladores del movimiento humano (flechas negras), susceptibles de neurorehabilitación (NR).

Vol. 59(1): 52 - 662018 
en las respuestas motoras a los estímulos somatosensoriales $(14,25)$, pérdida de equilibrio y aumento de riesgo a caídas $(20,26)$. Estos hechos impactan la calidad de vida de los adultos mayores y su interacción social con la comunidad (27). Así mismo, disminuye la independencia funcional durante el envejecimiento (28). Además, estos hechos generan discapacidad y en ocasiones, la muerte $(28,29)$.

Por lo anterior, se deben analizar las características de marcha desde diferentes ángulos que permitan entender el control postural, el cual se redefine a cada instante del movimiento corporal. Esto incluye la integración del centro de masa del cuerpo, la base de apoyo, la interacción entre la fuerza del individuo al desplazarse y la acción de la gravedad en el cuerpo del adulto mayor (30).

La integración neurofisiológica coordina los diversos mecanismos del movimiento mencionados, logrando una interacción que facilita el movimiento coordinado de la marcha o desplazamiento. De igual manera, la distancia que recorra el adulto mayor, dependerá es de la capacidad motora y neurológica que el individuo posee, las que se reducen en el adulto mayor (31); sin embargo, no se ha esclarecido por completo la razón de la reducción de la actividad de locomoción (32).

De manera limitada se sabe que la edad, el género y el desempeño del individuo durante las actividades de la vida diaria, así como el deterioro sistémico por envejecimiento normal, son elementos que modifican el patrón de marcha del adulto mayor $(33,34)$. El deterioro y la disminución de la velocidad de la marcha se han asociado también a reducción de la fuerza muscular; este último hecho se considera una modificación corporal "natural" del envejecimiento (35-37).

El declive de la función de los sistemas corporales del adulto mayor genera una fragilidad progresiva, lo que afecta la capacidad de adaptación del individuo que envejece. Estos hechos limitan la deambulación independiente en el adulto mayor, con un incremento de la morbimortalidad debido al sedentarismo $(38,39)$. Por ejemplo, la alteración de la marcha en adultos mayores se ha asociado con un mayor riesgo de caídas (40). De manera interesante se ha encontrado que cambios atróficos del cuerpo calloso (41) y de la corteza cerebral motora, se asocian con los cambios en la marcha en la población geriátrica $(42,43)$. Estos cambios morfológicos cerebrales coinciden con la degeneración de ganglios basales (44), trastornos del equilibrio y del control motor fino relacionado con la marcha $(34,45)$. En otros casos, se ha encontrado asociación entre la atrofia del hipocampo y la disminución de la velocidad y la longitud del paso en adultos mayores sanos (35). Dicha alteración de la velocidad se asocia, en algunos casos, con el deterioro de la función cerebral sensorial (36), que se refleja en los cambios del ciclo de desplazamiento y oscilación de la marcha y la disminución de la longitud del paso (37).

Por lo anterior, el adulto mayor debe realizar adaptaciones compensatorias dentro del patrón de marcha (38), con fases más cortas $(39,40)$, así como aumento del ancho de paso y el doble apoyo prolongado. Por esto, con la edad surgen estrategias compensatorias destinadas a aumentar la estabilidad, evitar caídas, y/o reducir el costo energético que genera la movilidad (41).

\section{ASPECTOS NEUROBIOLÓGICOS}

La pérdida de neuronas en el locus coeruleus se correlaciona con la reducción del aprendizaje motor $(42,43)$. Así mismo, el nivel de neurotransmisores se correlaciona con el comportamiento motor $(44,45)$. Después de los 65 años de vida, los receptores dopaminérgicos decrecen su función hasta en un $10 \%$, en individuos normales (43). De 
manera interesante, la perdida de equilibrio en el adulto mayor se asocia con la disminución de los niveles de dopamina. Específicamente, la reducción de dopamina en la zona del estriado se ha encontrado como predictor de caídas $(44,45)$. Otros estudios demuestran una asociación entre los niveles bajos de dopamina, la disminución de la velocidad de la marcha y la duración del doble apoyo y apoyo unipodal en los adultos mayores (46).

La denervación neuronal que se origina por patologías neurodegenerativas, afecta al sistema dopaminérgico (47) y la actividad de receptores de dopamina tipo D1 y D2. La zona nigroestriatal responsable de la realización de movimientos finos, (48) se encuentra alterada en la enfermedad de Parkinson (49), una entidad caracterizada por trastornos de la marcha, asociada con alteración de receptores dopaminérgicos. De manera interesante, las alteraciones de los receptores de dopamina, alteran a su vez las funciones superiores cognitivas que ejecutan el trabajo motor (50). Por lo anterior, se plantea que la motivación puede ayudar a modular la marcha en el adulto mayor $(51,52)$. Cabe resaltar que el estudio HAROLD por sus siglas en inglés (Modelo de la Reducción de la Asimetría del Hemisferio en Adultos Mayores), demostró que los adultos mayores presentan una disminución de la lateralización de la corteza prefrontal, una estructura clave para realizar tareas cognitivas.

Algunos estudios también han demostrado que el cerebro del adulto mayor realiza ajustes muy sutiles e imperceptibles que, en algunos casos, son iguales a los realizados por individuos más jóvenes durante la ejecución de tareas motoras (53-55). Otros estudios (56) han documentado diferencias relacionadas con el reclutamiento neuronal durante la actividad de ejecución de tareas motoras en edad adulta (57). El reclutamiento neuronal no selectivo de la actividad motora (58) requiere la acción de áreas cerebrales diferentes a la motora, lo cual compensa la actividad motora alterada; esto hace que la marcha sea menos precisa con el paso de los años, además de lo mencionado (59). Estos hallazgos, sin embargo, no se correlacionan con el nivel de demandas de la acción motora, ni refleja la reorganización o compensación neurobiológica propia del envejecimientonormal (60). Parece ser que los sistemas prefrontales que modulan procesos cognitivos son los más vulnerables a las pérdidas relacionadas con la edad, lo que reduce la disponibilidad de mecanismos de compensación. Para llegar a comprender, en su totalidad, los mecanismos neurobiológicos involucrados en la marcha que se ejecuta durante el envejecimiento normal, es indudable que se deben realizar más estudios.

\section{ASPECTOS DE LABORATORIO Y CONTROL MOTOR}

El estudio de la marcha humana permite conocer las deficiencias existentes en los sistemas involucrados $\mathrm{y}$, en consecuencia, planear de manera específica, los programas de neuromodulación y neurorehabilitación (61). Hay que tener en cuenta que las medidas obtenidas del análisis de marcha fluctúan, incluso cuando los diferentes factores de confusión se controlan de manera estricta, medidos por métodos tan rigurosos como fractales.

Las respuestas motoras de marcha ocurren como una sucesión de actividades que se originan en las aferencias periféricas como la retina, los receptores laberínticos y propioceptivos. Estos impulsos neurales actúan de manera sincrónica para generar equilibrio y estabilidad durante el desplazamiento del cuerpo. Además, existe control inter e intra segmental, así como modulación especializada de dicho control por parte de estructuras ubicadas a nivel cerebral y cerebelar.

En el estudio de la marcha, se deberá incluir la relación entre el control motor y los factores 
Bayona-Prieto y col.

biológicos que por envejecimiento alteran la marcha. Dentro de estos se destacan la atrofia neuronal normal que se asocia a la pérdida de equilibrio, enlentecimiento de movimiento, déficits de coordinación y de la marcha. Estos hechos alteran respuestas neurofisiológicas involucradas en el control motor fino y grueso (64).

Trabajos realizados en el siglo pasado se centraron, principalmente, en investigar la participación de mecanismos periféricos en la marcha del adulto mayor. Dentro de estos estudios, se destacan los hechos alrededor de la función de los receptores periféricos de superficie, así como la modulación de respuestas musculares y articulares (17-25,65). En la última década, la atención se ha desplazado al estudio de los mecanismos que interactúan en el sistema nervioso central $(66,67)$, y como las diversas estructuras encefálicas logran facilitar los procesamientos de la información sensorio-motora. Estos hallazgos han permitido incrementar el conocimiento del control motor en el adulto mayor $(68,69)$.

En adulto mayor, el envejecimiento de la integración sensorimotora aumenta el riesgo de caídas (68). Estos hechos originan enlentecimiento en la ejecución de tareas y deficiencia de coordinación (40), lo que incrementa las alteraciones en la velocidad de su marcha $(69,70)$. Además, hay alteraciones biomecánicas con limitación de la movilidad $(40,71)$. De la misma forma, se han observado déficits de rendimiento temporo-espacial, al no poder realizar acciones motoras que involucre al menos dos articulaciones $(70,71)$. Estos aspectos deberán ser tenidos en cuenta en estudios futuros que investiguen la marcha en el adulto mayor, para predecir complicaciones biomédicas asociadas con la fragilidad, la discapacidad, las caídas, y aun la muerte prematura (72).

En la actualidad se ha implementado el análisis de marcha usando diferentes aditamentos, que incluyen cámaras de video. Esto facilita el análisis de los diversos patrones cinéticos y cinemáticos del movimiento humano (73). Los datos obtenidos se usan para inducir la bioretroalimentación (74) y realizar la descripción cuantitativa del movimiento, con el fin de ayudar a construir nuevos engramas de reacondicionamiento $(75)$.

\section{NEUROREHABILITACIÓN Y NEURORESTAURACIÓN}

El enlentecimiento de la marcha que ocurre con el paso de los años, hace que el adulto mayor dude si puede recuperar o evitar el deterioro de su locomoción. La neurorehabilitación puede mejorar estas deficiencias $(76,77)$, utilizando diversos mecanismos de re-aprendizaje del movimiento humano (Fig. 2).

Para lograr este objetivo se requiere desarrollar una mejor comprensión de la neurorehabilitación y neurorestauración $(78,79)$. Dentro de estos aspectos se destaca el hecho de que las estructuras relacionadas con la marcha, son susceptibles de modulación plástica, lo cual ayuda a disminuir la restricción funcional de la marcha (79). Así mismo, una mejor comprensión de la relación entre el sistema motor y cognitivo podría ser útil para diseñar mejores estrategias de neurorestauración integral. Esto debido a que el deterioro cognitivo influye la disminución de la velocidad de la marcha, siendo este un predictor objetivo de discapacidad física $(80,81)$. Por tal motivo se deben analizar en cada caso los planes de rehabilitación personalizada, en los cuales se integren el ejercicio, el entrenamiento motor, la rehabilitación cognitiva, el empleo de agentes no farmacéuticos y los dispositivos bioelectrónicos, con el fin de ayudar al reaprendizaje de la marcha.

En los adultos mayores se puede implementar un número de intervenciones no invasivas (82). Por ejemplo, la retroalimentación promueve 
mejoría en el patrón de movimiento del individuo (83). La biorretroalimentación, de otro lado, requiere que los sistemas indemnes, pero inactivos, se activen para ejecutar las respuestas esperadas, incluyendo reaprendizaje del control postural (72). En este sentido, se observó un mayor reclutamiento de actividad sináptica en áreas corticales y subcorticales usualmente no activadas, comparadas con las áreas activadas en individuos jóvenes (84).

La neurorehabilitación re-direcciona las tareas en tiempo definido. Lo anterior se basa en la implementación de los modelos terapéuticos transdiciplinares, los cuales logra que el adulto mayor re-direccione la capacidad de realizar el movimiento casi al mismo nivel que un adulto joven. Lo anterior se soporta en estudios que indican que los adultos mayores crean nuevos niveles de sinapsis en zonas como las cortezas parietal, premotora, prefrontal izquierda y cingulada anterior; cabe anotar que adultos jóvenes expuestos a condiciones similares solo reclutaron regiones motoras (74-77). Esto indica que los adultos mayores reaprenden a hacer tareas motoras y los lleva a reclutar zonas adicionales en el cerebro, mientras los jóvenes para realizar la misma actividad no requieren una activación de nuevas zonas cerebrales (71).

Heuninckx y col. (85) concluyeron que los adultos mayores al ser sometidos a entrenamiento motor, activan zonas cerebrales asociadas a procesos ejecutivos de tipo cognitivo (86), mientras que los adultos jóvenes realizaban los procesos motores con zonas cerebrales automatizadas (71-77). Estos hallazgos en los adultos mayores se asocian a activación cortical de regiones premotoras y suplementarias motoras, las cuales están asociadas a procesos cognitivos (87). Se sugiere, entonces, que los programas de neurorehabilitación involucren planes de reaprendizaje y plasticidad para que las áreas motoras suplementarias incrementen la interacción sináptica en zonas como la corteza sensoriomotora y premotora ventral (88), a nivel bilateral (72-89). Cabe resaltar que los diversos procedimientos y estrategias (79) de neurorehabilitación que promueven plasticidad neuronal en adultos mayores (74-82) son procedimientos no invasivos y seguros.

\section{CONCLUSION}

Estudios relacionados con la marcha del adulto mayor y su neurofisiatria, deberán ser una prioridad de investigación neurobiológica mundial. En épocas recientes, la relación entre la neurobiología funcional y el control sensorimotor ha logrado que se comprenda de una mejor forma, la integración sensorial y sus efectos en la marcha del adulto mayor. Los sistemas de procesamiento de imágenes y análisis de movimiento como el SIMI, entre otros, ofrecen una retroalimentación cuantificable de la función y disfunción de la marcha del adulto. Esta información es un aspecto importante para ayudar a implementar la neurorehabilitación y neurorestauración del movimiento en el adulto mayor. El uso apropiado de herramientas holísticas en neurorehabilitación y neurología restaurativa ayudarán a modular, de una mejor forma, el re-aprendizaje de la marcha en adultos mayores, con el fin de mejorar la calidad de vida de este grupo poblacional. Independiente de los aspectos mencionados, se deberá motivar al adulto mayor a realizar ejercicio bajo orientación profesional. Esto debido a que el ejercicio modula un número de funciones corporales, las cuales impactan de manera favorable la calidad de vida de los adultos mayores.

\section{REFERENCIAS}

1. UNFPA, HelpAge International. Envejecimiento en el Siglo XXI: Una Celebración y un Desafío. Fondo de 


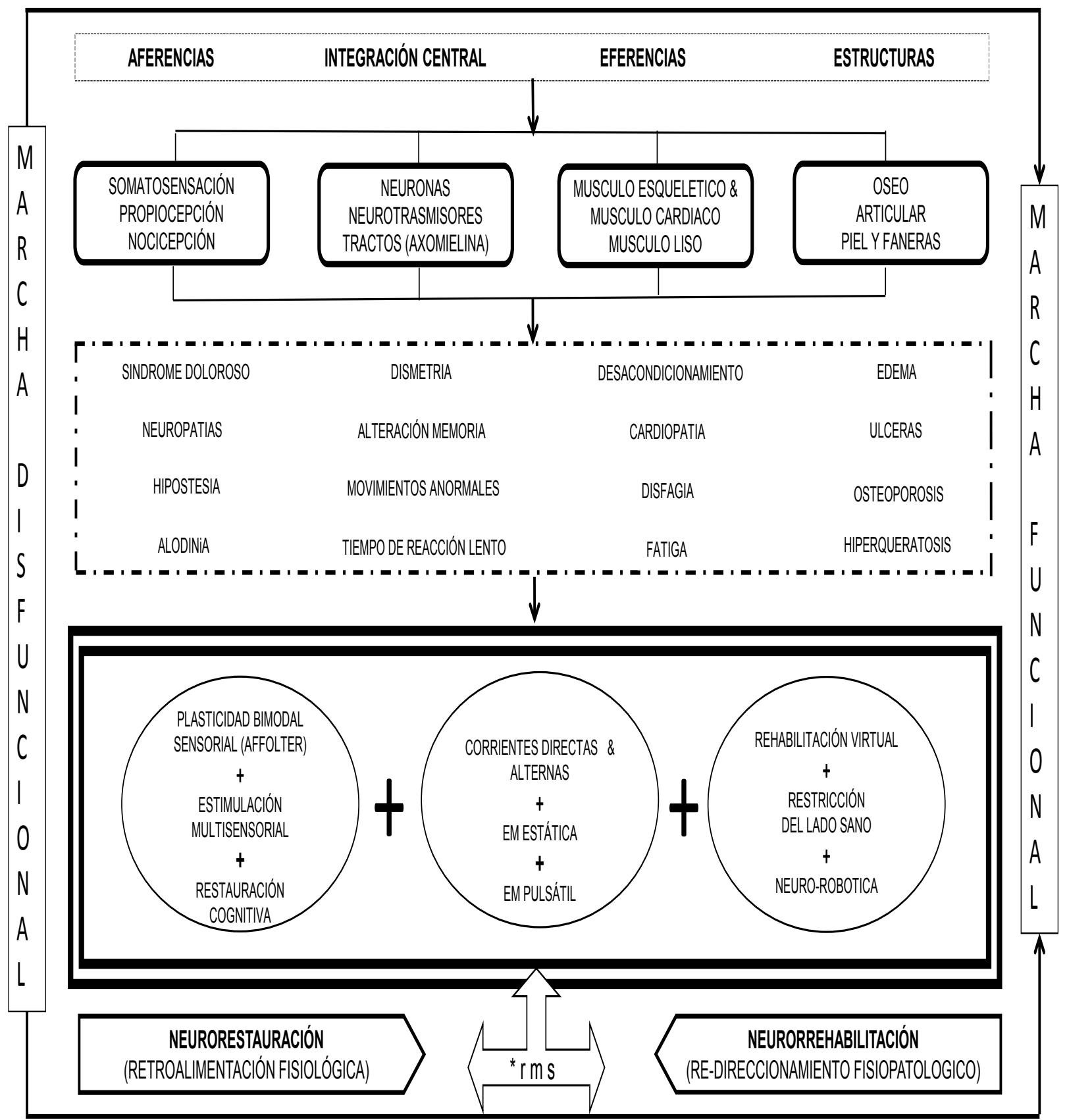

Fig.2. Modelo neurofisiatrico de la marcha senil. Sistemas (línea de puntos), funciones (rectángulo) y tastornos (línea de rayas y puntos) de la marcha. Reaprendizaje sensorimotor (línea doble) de la marcha inducido por la neuroplasticidad resultante de la neurorestauración y la neurorrehabilitación. * : análisis computarizado de la marcha (ejemplo: r. m. s. : reality motion system SIMI ${ }^{\circledR}$ 
Población de Naciones Unidas UNFPA [on line] 2012 Oct [citado 2016 Mar 06]; [190 pagina]. Disponible en: https://www.unfpa. org/sites/default/files/pubpdf/Ageing\%20 Report $\% 20$ Executive\%20Summary $\% 20$ SPANISH\%20Final_0.pdf

2. Organización Mündial de la Salud. Informe Mundial sobre el envejecimiento y la salud. OMS [on line] 2015 May [citado 2016 Mar 5]; [282 paginas]. Disponible en: http://apps.who.int/iris/ itstream/10665/186466/1/9789240694873 spa.pdf

3. Bake R. Gait analysis methods in rehabilitation. J Neuroeng Rehabil 2006; 3:4-10.

4. Morreno de La Fuente JL. Podología General y Biomecánica. 2da Ed. Barcelona: Masson; 2009, p 334.

5. Corujo RE, Pérez HD. Cambios más relevantes y peculiaridades de las enfermedades en el anciano. En: Sociedad Española de gerontología Eds. Tratado de Geriatría para residentes. Madrid: International Marketing \& Communication; 2006, P 47-58.

6. Bloem Br, Haan J, Lagaay A, Bek W, Wintzen A, Raymund A. Investigation of Gait in elderly subjects over 88 years of age. J Geriatr Psychiatry Neurol 1992; 5:78-84.

7. Ministerio de la Protección Social. República de Colombia: Política nacional de envejecimiento y vejez. Bogotá: Imprenta Nacional; 2007, p 50.

8. Albala C, Lebrão ML, León Díaz EM, Ham-Chande R, Hennis AJ, Palloni A, Peláez M, Pratts O. Encuesta Salud, Bienestar y Envejecimiento (SABE): metodología de la encuesta y perfil de la población estudiada. Rev Panam Salud Publica 2005;17:307-22.

9. Forsell C, Conradsson D, Paquette C, Franzén E. Reducing gait speed affects axial coordination of walking turns. Gait Posture 2017; 54:71-75.

10. Verghese J, LeValley A, Hall CB, Katz MJ, Ambrose AF, Lipton RB. Epidemiology of gait disorders in community-residing older adults. J Am Geriatr Soc 2006;54:255-261.

11. Viladot Voegeli A. Marcha humana. En: Nuñez-Samper M, Llanos Alcanzar LF. Eds. Biomecánica, medicina y cirugía del pie. Barcelona: Masson; 1997, P 82-89.

12. Koller WC, Wilson RS,Glatt Sl, Huckman MS, Fox JH. Senile gait: correlation with computer tomographic scans. Ann Neurol $1983 ; 13: 343-344$.

13. Maki BE. Gait changes in older adults: predictors of falls or indicators of fear. J Am Geriatr Soc 1997; 45: 313-320.

14. Alexander RM. Bipedal animals, and their differences from humans. J Anat 2004; 204:321-330.

15. D de Bruin E, Schmidt A. Walking behaviour of healthy elderly: attention should be paid. Behav Cerebro Funct 2010; 6:2-8.

16. Pamoukdjian F, Paillaud E, Zelek L, Laurent M, Lévy V, Landre T, Sebbane G. Measurement of gait speed in older adults to identify complications associated with frailty: A systematic review. J Geriatr Oncol 2015; 6:484-496.

17. Sudarsky L. Gait disorders: prevalence, morbidity, and etiology. Adv Neurol 2001; 87:111-117.

18. Latash ML, Scholz JP, Schöner G. Toward a new theory of motor synergies. Motor Control 2007; 11:276-308.

19. Martín-Lesende I, López-Torres JD, deHoyos MC, Baena JM, Gorroñogoitia A, Herreros Y. Actividades preventivas en los mayores. Atención Primaria 2014; 46:111117.

20. Lluis Ramos GE, Llibre Rodríguez JJ. Fragilidad en el adulto mayor: Un primer

Vol. 59(1): 52 - 662018 
acercamiento. Rev Cubana Med Gen Integr [on line] 2004 Jan-Mar [citado 2016 Ago 8]. Disponible en: http://scielo.sld.cu/ scielo.php?script $=$ sci_arttext\&pid $=\mathrm{S} 0864$ $21252004000400009 \& \operatorname{lng}=\mathrm{es} \& n \mathrm{~nm}=\mathrm{iso}$

21. González Ramírez $A$, Lázaro del Nogal M, Ribera Casado JM. Valoración de los sistemas de control postural en ancianos con caídas de repetición Rev Esp Geriatr Gerontol 2008; 43:71-75.

22. Hausdorff JM, Peng CK, Goldberger AL, Stoll AL. Gait unsteadiness and fall risk in two affective disorders: a preliminary study. BMC Psychiatry 2004; 4:1-7.

23. Belló M, Puentes-Rosas E, Medina-Mora ME, Lozano R. Prevalencia y diagnóstico de depresión en población adulta en México. Salud Pública Mex 2005; 47: S4-S11.

24. Guralnik JM, Fried LP, Salive ME. Disability as a public health outcome in the aging population. Annu Rev Public Health 1996; 17:25-46.

25. Mendes-Chiloff CL, Ramos-Cerqueira AT, Pereira MC, Rodriguez A. Depressive symptoms among elderly inpatients of a Brazilian university hospital: prevalence and associated factors. Int Psychogeriatr 2008; 20:1028-1040.

26. Hoyl MT, Alessi CA, Harker JO, Josephson KR, Pietruszka FM, Koelfgen M. Development and testing of a five-item version of the Geriatric Depression Scale. J Am Geriatr Soc 1999; 47: 873-878.

27. Alfieri FM, Riberto M, Lopes JA, Filippo TR, Imamura M, Battistella LR. Postural control of healthy elderly individuals compared to elderly individuals with stroke sequelae. Open Neurol J 2016; 10:1-8.

28. Alfonso Silguero SA, Martínez-Reig M, Gómez Arnedo L, Juncos Martínez G, Romero Rizos L, Abizanda Soler P. Enfermedad crónica, mortalidad y discapacidad en una población española de edad avanzada: el estudio FRADEA. Rev Esp Geriatr Gerontol 2014; 49: 51-58.

29. Fasano A, Bloem BR. Gait disorders. Continuum 2013; 19:1344-1382.

30. Guralnik JM, Ferrucci L, Pieper CF, Leveille SG, Markides KS, Ostir GV, Studenski S, Berkman LF, Wallece RB. Lower extremity function and subsequent disability consistency across studies, predictive models, and value of gait speed alone compared with the short physical performance battery. J Gerontol A Biol Sci Med Sci 2000; 55:M221-M231.

31. Jernigan TL, Archibald SL, FennemaNotestine CH, Gamst AC, Stout JC, Bonner J, Hesselink JR. Effects of age on tissues and regions of the cerebrum and cerebellum. Neurobiol Aging 2001; 22:581594.

32. Seidler RD, Bernard JA, Burutolu TB, Fling BW, Gordon MT, Gwin JT, Kwak Y, Lipps DB. Motor control and aging: links to age-related brain structural, functional, and biochemical effects. Neurosci Biobehav Rev 2010; 34:721-733.

33. Courchesne E, Chisum HJ,Townsend J, Cowles A, Covington J, Egaas B, Harwood M, Hinds S, Press GA. Normal brain development and aging: quantitative analysis at in vivo MR imaging in healthy volunteers. Radiology 2000; 216:672-682.

34. Ge Y, Grossman RI, Babb JS, Rabin M, Mannon L, Kolson L. Age-related total gray matter and white matter changes in normal adult brain. Part I: volumetric MR imaging analysis. Am J Neuroradiol 2002; 23:1327-1333.

35. Callisaya ML, Beare R, Phan TG, de Blizzard L, Thrift AG, Chen J, Srikanth VK. Brain structural change and gait decline: a longitudinal population-based study. J Am Geriatr Soc 2013; 61:1074-1079.

36. Raz N, Rodrigue KM, Kennedy KM, 
Head D, Gunning-Dixon F, Acker JD. Differential aging of the human striatum: longitudinal evidence. AJNR Am J Neuroradiol 2003; 24:1849-1856.

37. Kirkwood RN, de Souza Moreira B, Vallone ML, Mingoti SA, Dias RC, Sampaio RF. Step length appears to be a strong discriminant gait parameter for elderly females highly concerned about falls: a cross-sectional observational study. Physiotherapy 2011; 97:126-131.

38. Polcyn AF, Lipsitz LA, Kerrigan DC, Collins JJ. Age-related changes in the initiation of gait: degradation of central mechanisms for momentum generation. Arch Phys Med Rehabil 1998; 79:15821589.

39. Lord SR, Lloyd DG, LI SK. Sensorimotor function, gait patterns and falls in community-dwelling women. Age Ageing 1996; 25:292-299.

40. Cutlip RG, Mancinelli C, Huber F, DiPasquale J. Evaluation of an instrumented walkway for measurement of the kinematic parameters of gait. Gait Posture 2000; 12:134-138.

41. Pavol MJ, Owings TM, Foley KT, Grabiner MD. Gait characteristics as risk factors for falling from trips induced in older adults. J Gerontol Ser A Biol Sci Med Sci 1999; 54:M583-M590.

42. Gould TJ, Bickford PC. The effects of aging on cerebellar beta-adrenergic receptor activation and motor learning in female F344 rats. Neurosci Lett 1996; 216:53-56.

43. Kaasinen V, Vilkman $H$, Hietala J, Någren K, Helenius H, Olsson H, Farde L, Rinne J. Age-related dopamine D2/D3 receptor loss in extrastriatal regions of the human brain. Neurobiol Aging 2000; 21:683-688.

44. Cham R, Perera S, Studenski SA, Bohnen NI. Striatal dopamine denervation and sensory integration for balance in middle- aged and older adults. Gait Posture 2007; 26:516-525.

45. Cham R, Studenski SA, Perera S, Bohnen NI. Striatal dopaminergic denervation and gait in healthy adults. Exp Brain Res 2008; 185:391-398.

46. Floel A, Vomhof P, Lorenzen A, Roesser N, Breitenstein C, Knecht S. Levodopa improves skilled hand functions in the elderly. Eur J Neurosci 2008; 27:13011307.

47. Kaasinen V, Rinne JO. Functional imaging studies of dopamine system and cognition in normal aging and Parkinson's disease. Neurosci Biobehav Rev 2002; 26:785-793.

48. Van Dyck Ch, Avery RA, MacAvoy MG, Marek KL, Quinlan DM, Baldwin RM, Seibyl J, Innis RB, Arnsten AF. Striatal dopamine transporters correlate with simple reaction time in elderly subjects. Neurobiol Aging 2008; 29: 1237-1246.

49. Volkow ND, Logan J, Fowler JS, Wang GJ, Gur RC, Wong Ch, Felder Ch, Gatley J, Ding Y, Hitzemann R, Pappas N. Association between age-related decline in brain dopamine activity and impairment in frontal and cingulate metabolism. Am J Psychiatry 2000; 157:75-80.

50. Arnsten AF, Li BM. Neurobiology of executive functions: catecholamine influences on prefrontal cortical functions. Biol Psychiatry 2005; 57:1377-1384.

51. Bonin-Guillaume S, Hasbroucq T, Blin O. Psychomotor retardation associated to depression differs from that of normal aging. Psychol Neuropsychiatr Vieil 2008; 6:137-144.

52. Bo J. Seidler DR. Visuospatial working memory capacity predicts the organization of acquired explicit motor sequences. J Neurophysiol 2009; 1: 3116-3125.

53. Calautti C, Serrati C, Baron JC. Effects of age on brain activation during auditory-

Vol. 59(1): 52 - 662018 
cued thumb-to-index opposition: A positron emission tomography study. Stroke 2001; 32:139-146.

54. Heuninckx S, Wenderoth N, Swinnen SP. Systems neuroplasticity in the aging brain: recruiting additional neural resources for successful motor performance in elderly persons. J Neurosci 2008; 28:91-99.

55. Mattay VS, Fera F, Tessitore A, Hariri AR, Das S, Callicott JH, Weinberger DR. Neurophysiological correlates of agerelated changes in human motor function. Neurology 2002; 58:630-635.

56. Mihara M, Miyai I, Hatakenaka M, Kubota K, Sakoda S. Role of the prefrontal cortex in human balance control. Neuroimage 2008; 43:329-336.

57. Cabeza R. Cognitive neuroscience of aging: contributions of functional neuroimaging. Scand J Psychol 2001; 42:277-286.

58. Riecker A, Gröschel K, Ackermann H, Steinbrink C, Witte O, Kastrup A. Functional significance of age-related differences in motor activation patterns. Neuroimage 2006; 32:1345-1354.

59. Wu T, Hallett M. The influence of normal human ageing on automatic movements. J Physiol 2005; 562:605-615.

60. Tecchio F, Zappasodi F, Pasqualetti P, De Gennaro L, Pellicciari MC, Ercolani M. Age dependence of primary motor cortex plasticity induced by paired associative stimulation. Clin Neurophysiol 2008; 119:675-682.

61. Bayona EA, Bayona-Prieto J, LeonSarmiento FE. Neuroplasticity, neuromodulation, and neurorehabilitation: Three different concepts, one only true goal. Salud Uninorte 2011; 27: 95-107.

62. Goble DJ, Marino GW, Potvin JR. The influence of horizontal velocity on interlimb symmetry in normal walking. Hum Mov Sci 2003; 22: 271-283.
63. Hartmanm A, Murer K, Bie R, Bruin E. Reproducibility of spatio-temporal gait parameters under different conditions in older adults using a trunk triaxial accelerometer system. Gait Posture 2009;30: 351-355.

64. León Sarmiento FE, Bayona Prieto J, Bayona E. Reflexología, nocicepción y control motor. Revista IATREIA. 2005; 18:4-5.

65. Lindenberger $U$, Marsiske $M$, Baltes PB. Memorizing while walking: increase in dual-task costs from young adulthood to old age. Psychol Aging 2000; 15:417-436.

66. Kristinsdottir EK, Fransson PA, Magnusson M. Changes in postural control in healthy elderly subjects are related to vibration sensation, vision and vestibular asymmetry. Acta Otolaryngol 2001; 121:700-706.

67. Bacsi AM, Colebatch JG. Evidence for reflex and perceptual vestibular contributions to postural control. Exp Brain Res 2005; 160:22-28.

68. Harada T, Miyai I, Suzuki M, Kubota K. Gait capacity affects cortical activation patterns related to speed control in the elderly. Exp Brain Res 2009; 193:445-454.

69. Contreras-Vidal JL, Teulings HL, Stelmach GE. Elderly subjects are impaired in spatial coordination in fine motor control. Acta Psychol (Amst) 1998; 100:25-35.

70. Wishart LR, Lee TD, Murdoch JE, Hodges NJ. Effects of aging on automatic and effortful processes in bimanual coordination. J Gerontol B Psychol Sci Soc Sci 2000;55: 85-94.

71. Stelmach GE, Populin L, Müller F. Postural muscle onset and voluntary movement in the elderly. Neurosci Lett 1990; 117:188-193.

72. Van der Ploeg E, Van der Velde Y, Gobbens R. Predictive Value of Gait Speed and frailty indicators on survival time and time until discharge to home in ambulant 
older patients in a specialized rehabilitation center. J Geriatr Phys Ther 2016:4:1-7.

73. Perera S, Patel KV, Rosano C, Rubin SM, Satterfield S, Harris T, Ensrud K, Orwoll E, Lee CG, Chandler JM, Newman AB, Cauley JA, Guralnik JM, Ferruci L, Studenski SA. Gait speed predicts incident disability: A Pooled Analysis. J Gerontol A Biol Sci Med Sci 2016; 71:63-71.

74. Milner CE, Paquette MR. A kinematic method to detect foot contact during running for all foot strike patterns. J Biomech 2015; 48:3502-3505.

75. Watson NL, Rosano C, Boudreau RM, Simonsick EM, Ferrucci L, SuttonTyrrell K, Hardy S, Atkinson H, Yaffe K, Satterfield S, Harris T, Newman A. Executive function, memory, and gait speed decline in well-functioning older adults. J Gerontol A: Biol Sci Med Sci 2010; 65:1093-1100.

76. Sweeting K, Mock M. Gait Posture. Assessment in general practice. Austral Fam Physic 2007;36: 398-405.

77. Busch TA, Duarte YA, Pires Nunes D, Lebrão ML, Amaro E Jr, Naslasky MS, Rodrigues AD. Factors associated with lower gait speed among the elderly living in a developing country: a cross-sectional population-based study. BMC Geriatr 2015; $1: 15-35$.

78. Leon-Sarmiento FE, Bayona- Prieto J, Yuli Cadena. Plasticidad neuronal, neurorehabilitación y trastornos del movimiento: el cambio es ahora. Acta Neurol Colomb 2008; 24:40-42.

79. Leon-Ariza DS, Leon-Ariza JS, Bayona EA, Bayona-Prieto J, Leon-Sarmiento FE. Remote dysfunctions in spinal cord injury: Closer than ever. Clin Neurol Neurosurg 2015; 133:102-103.

80. Vereecke EE, D'Août K, Aerts P. The dynamics of hylobatid bipedalism: evidence for an energy-saving mechanism? J Exp Biol 2006; 209:2829-2838.

81. Di Domizio DP. Aprendizaje motor en adultos mayores. [Tesis Doctoral] Buenos Aires: Universidad Nacional de la Plata; 2004.

82. Cho SH, Park JM, Kwon OY. Gender differences in three-dimensional gait analysis data from 98 healthy Korean adults. Clin Biomech 2004; 19:145-152.

83. Tate JJ, Milner CE. Real-Time Kinematic, Temporospatial, and kinetic biofeedback during gait retraining in patients: A systematic review. Phys Ther 2010; 90: 1123-1134

84. Naccarato M, Calautti C, Jones PS, Day DJ, Carpenter TA, Baron JC. Does healthy aging affect the hemispheric activation balance during paced index-tothumb opposition task? An fMRI study. Neuroimage 2006; 32:1250-256

85. Park DC, Reuter-Lorenz P. The Adaptive Brain: Aging and neurocognitive scaffolding. Annu Rev Psychol 2009; 60: 173-196.

86. Heuninckx S, Wenderoth N, Debaere F, Peeters R, Swinnen SP. Neural basis of aging: the penetration of cognition into action control. J Neurosci 2005; 25:67876796.

87. Liepert $\mathbf{J}$, Classen $J$, Cohen LG, Hallett M. Task-dependent changes of intracortical inhibition. Exp Brain Res 1998; 118:421-426.

88. Li SC, Brehmer Y, Shing YL, Werkle-Bergner $M$, Lindenberger U. Neuromodulation of associative and organizational plasticity across the life span: empirical evidence and neurocomputational modeling. Neurosci Biobehav Rev 2006; 30:775-790.

89. Taniwaki T, Okayama A, Yoshiura T, Togao O, Nakamura Y, Yamasaki T,

Vol. 59(1): 52 - 662018 
Ogata K, Shigeto H, Ohyagi Y, Kira J, Tobimatsu S. Age-related alterations of the functional interactions within the basal ganglia and cerebellar motor loops in vivo. Neuroimage 2007; 36:1263-1276. 